\title{
Uso de bicarbonato e lactato-L para correção da acidose metabólica sistêmica em bovinos com acidose láctica ruminal aguda
}

\author{
[Use of bicarbonate and lactate L for correction of systemic metabolic acidosis in cattle \\ with acute rumen lactic acidosis] \\ M.L.R. Leal ${ }^{1}$, C.A. Maruta ${ }^{2}$, E.L. Ortolani ${ }^{2 *}$ \\ ${ }^{1}$ Universidade Federal de Santa Maria - Santa Maria, RS \\ ${ }^{2}$ Faculdade de Medicina Veterinária e Zootecnia - USP \\ Av. Prof. Orlando Marques de Paiva, 87, \\ 05508-900 - São Paulo, SP
}

\begin{abstract}
RESUMO
Foram utilizados seis novilhos, providos de cânula ruminal, em delineamento experimental cross-over, para comparar a eficiência de soluções de bicarbonato de sódio e lactato-L de sódio na correção da acidose metabólica sistêmica (AMS), causada pela acidose láctica ruminal (ALR). Vinte horas após, quando apresentavam intensa AMS, os animais foram distribuídos aleatoriamente e tratados com cinco litros de $150 \mathrm{mMol} / 1$ de bicarbonato de sódio ou de lactato-L de sódio, infundidas por via intravenosa, nas quatro horas seguintes. Amostras de sangue, para hemogasometria, foram coletadas no decorrer da infusão a zero, 1, 2, 3, 4, 6 e 8 horas. Ambos os tratamentos elevaram o pH sangüíneo já na primeira hora pós-infusão, corrigindo adequadamente a AMS. O tratamento com lactato-L de sódio aumentou as concentrações de bicarbonato, $\mathrm{TCO}_{2}$ e EAB sangüíneos já na segunda hora pós-infusão; com o bicarbonato essa elevação ocorreu a partir da terceira hora. Não houve diferenças entre tratamentos para $\mathrm{pH}$ sangüíneo, bicarbonato, $\mathrm{TCO}_{2}$ e excesso de base. Vinte e quatro horas após o tratamento todos os novilhos apresentaram plena recuperação. O lactato-L pode substituir o bicarbonato na correção da AMS em novilhos com ALR.
\end{abstract}

Palavras-chave: bovino, acidose, bicarbonato, lactato-L

\begin{abstract}
The efficiency of sodium bicarbonate or l-lactate for correcting systemic metabolic acidosis (SMA) caused by rumen lactic acidosis (RLA) was evaluated using six rumen-cannulated steers in a cross-over experimental design. RLA was induced by administration of sucrose, intraruminally. Twenty hours later when the animals developed an intense SMA, the steers were randomly distributed and treated intravenously either with $5 \mathrm{l}$ of $15 \mathrm{mMol} / \mathrm{l}$ sodium bicarbonate or L-lactate solution, infused throughout 4h. Blood samples were colleted throughout the infusion at zero, 1, 2, 3, 4, 6 and 8h, for blood gas analysis. After Ihour, both sodium bicarbonate and L-lactate solutions increased blood $\mathrm{pH}$ and corrected adequately the SMA. Blood bicarbonate, $\mathrm{TCO}_{2}$ and base excess concentrations were also increased at the $2^{\text {nd }}$ hour with L-lactate and at the $3^{\text {rd }}$ hour with bicarbonate. No differences between treatments were observed for blood $\mathrm{pH}$, bicarbonate, $\mathrm{TCO}_{2}$ and base excess concentrations. Treated steers after twenty-four hours showed an effective clinical recovery. L-lactate can adequately replace bicarbonate in the correction of SMA in steers with RLA.
\end{abstract}

Keywords: cattle, acidosis, bicarbonate, lactate- $L$

Recebido em 16 de outubro de 2006

Aceito em 4 de junho de 2007

*Autor para correspondência (corresponding author)

E-mail: ortolani@usp.br 


\section{INTRODUÇÃO}

A pecuária bovina brasileira se tem modificado nas últimas décadas, apresentando tendência à intensificação de seu sistema de produção. Hoje, estima-se que cerca de 6 milhões dos bovinos sejam criados em regime de confinamento ou semiconfinamento, sendo alimentados com dietas ricas em energia (Anuário..., 2003). Nesse sistema, caso não haja um adequado manejo é comum o surgimento de quadros de acidose láctica ruminal (ALR) aguda (Maruta e Ortolani, 2002).

Tal enfermidade é gerada pela ingestão súbita ou exagerada de carboidratos solúveis nãoestruturais, os quais, fermentados pelas bactérias ruminais, produzem grandes quantidades de ácido láctico, levando inicialmente à acidose ruminal e em seguida a uma acidose metabólica sistêmica e desidratação que comumente pode levar o animal à morte (Maruta e Ortolani, 2002; Radostits et al., 2002).

A correção da acidose metabólica sistêmica é sem dúvida o ponto vital do sucesso da terapia empregada para a ALR (Howard, 1981; Underwood, 1992; Radostits et al., 2002). Existe também um consenso em indicar unicamente o uso de bicarbonato na correção da acidose sistêmica na ALR. Contudo, o uso indevido ou excessivo desse tampão pode trazer sérios inconvenientes aos animais, tais como: alcalose sistêmica iatrogênica, edema cerebral e acidose paradoxal do fluido cerebroespinhal (Hartsfield et al., 1981). Uma alternativa para superar esse problema seria a utilização de certos tampões metabolizáveis, os quais quando oxidados ou convertidos em glicose consomem no processo íons $\mathrm{H}+$. Esses tampões podem ser infundidos em altas quantidades sem gerar problemas colaterais, pois o próprio organismo regularia sua utilização, evitando uma sobrecarga (Naylor e Forsyth, 1986). Num estudo anterior, empregando-se bovinos adultos sadios, comparou-se o efeito alcalinizante no sangue promovido pela infusão de igual quantidade de alguns desses tampões \{lactato-levógiro (L), acetato-L, propionato-L, lactato, L e dextrogiro (D) \} com o bicarbonato, concluindo-se que os melhores resultados foram obtidos igualmente com o lactato-L e o bicarbonato (Leal et al., 2006).
Esse último estudo forneceu dados básicos e estimulou a realização de um próximo que comparasse a eficácia desses melhores tampões no tratamento de acidose metabólica sistêmica estabelecida. Assim, o objetivo do presente trabalho foi o de avaliar a eficiência corretiva da acidose metabólica sistêmica por soluções de lactato-L ou bicarbonato em bovinos submetidos a quadro de ALR aguda.

\section{MATERAL E MÉTODOS}

Foram utilizados seis novilhos mestiços, sadios, com cerca de $200 \mathrm{~kg}$ de peso vivo. Dois meses antes foram implantadas cânulas ruminais nos animais, para facilitar a indução experimental e o tratamento da ALR. A quantidade de matéria seca (MS) oferecida era correspondente a 2,5\% do peso vivo, de uma dieta composta de $80 \%$ da MS de feno de capim coast-cross (Cynodon dactylon) e $20 \%$ de ração concentrada (80\% farelo de trigo; $20 \%$ farelo de soja) e suplementação mineral e água ad libitum. Os animais foram submetidos a jejum alimentar 12horas antes da indução da ALR. Após o término de cada indução os garrotes foram alimentados exclusivamente com feno por 10 dias, retornando em seguida à dieta basal por no mínimo 30 dias antes de novo ensaio.

Empregou-se o cross-over no delineamento experimental. Todos os animais foram submetidos à indução de ALR e, em seguida, distribuídos aleatoriamente em dois grupos de três novilhos: metade foi tratada com bicarbonato e metade com lactato-L. Na indução seguinte, os tratamentos se inverteram. $\mathrm{O}$ intervalo entre as induções foi de, no mínimo, 40 dias* . A indução de ALR foi realizada segundo protocolo desenvolvido por Ortolani (1995), administrando-se na cânula ruminal quantidade de sacarose, de acordo com o peso metabólico corrigido, descontando-se $15 \%$ desse total calculado.

Amostras de fluido ruminal foram coletadas do saco ventral posterior, na $21^{\mathrm{a}}$ hora após a indução, para análise de seu $\mathrm{pH}^{1}$. Apenas animais com $\mathrm{pH}$ de fluido ruminal inferior a 4,4 e o pH e concentração de bicarbonato sangüíneos * O experimento seguiu as normas preconizadas pelo Comitê
Local de Bioética.

${ }^{1}$ pHmetro DM-20 Digimed - São Paulo, Brasil 
inferiores a 7,25 e $20 \mathrm{mM} / 1$, respectivamente, foram incluídos no ensaio, para caracterizar tanto acidose ruminal como a sistêmica. Antes do início do tratamento foram implantados nos animais dois cateteres ${ }^{2}$ de polietileno nas veias jugulares esquerda e direita. No primeiro deles injetaram-se os tampões; o segundo destinou-se à coleta de sangue para análise hemogasométrica. O primeiro cateter foi retirado após quatro horas do experimento.

O tratamento consistiu de infusão intravenosa de 51 de soluções isomolares $(150 \mathrm{mmol} / \mathrm{l})$ de bicarbonato $(12,60 \mathrm{~g} / \mathrm{l})$ ou de lactato-L $(16,65 \mathrm{~g} / \mathrm{l})$, ambas na forma sódica, adicionados em água bidestilada. Os tratamentos seguiram essa seqüência: 21 da solução na primeira hora e $11 \mathrm{em}$ cada uma das três horas subseqüentes, semelhante ao protocolo realizado por Kasari e Naylor (1985) em bezerros com diarréia. A fim de manter o cateter de coleta sangüínea sob constante fluxo foi infundido neste, no decorrer do experimento, 11 de solução salina isotônica.

Em todos os novilhos ainda foi instituído um tratamento de suporte que consistia na retirada de oito litros de fluido ruminal, nas quatro horas de tratamento, e colocação de igual quantidade de solução salina isotônica neste órgão, associada à transfusão de dois litros de fluido rúmen, obtido naquele momento de outro bovino sadio.

Foram realizadas coletas de sangue no tempo basal, e às 1, 2, 3, 4, 6 e 8 horas durante o tratamento intravenoso. Essas amostras foram coletadas, em condições anaeróbias, com seringa contendo heparinato de sódio como anticoagulante, diretamente do cateter intravenoso. $\mathrm{O} \mathrm{pH}$, a concentração de bicarbonato, o excesso de acido-base sangüíneo $(\mathrm{EAB})$ e a pressão de $\mathrm{CO}_{2}$ foram determinados em aparelho de hemogasometria ${ }^{3}$, seguindo recomendações sugeridas por Sucupira e Ortolani (2003).

Os dados obtidos foram submetidos a análise de variância em que os tampões estudados representaram as parcelas e os tempos de coleta as subparcelas. As médias foram comparadas por diferença mínima significativa (DMS) utilizando t tabelado ponderado e fixando como nível de

${ }^{2} \mathrm{BD}$ - Intracath $(1,7 \mathrm{~mm} \times 30,5 \mathrm{~mm})$ - Juiz de Fora, Brasil ${ }^{3}$ AVL - São Paulo, Brasil. significância 5\% (Sampaio, 2002). O coeficiente de variação $\left(\mathrm{CV}_{\mathrm{b}}\right)$ também foi calculado relativo à instabilidade das subparcelas (Sampaio, 2002).

\section{RESULTADOS}

Em todos os casos e momentos o $\mathrm{pH}$ do fluido ruminal encontrou-se entre 4,37 e 4,0. Todos os novilhos apresentaram, no momento basal, $\mathrm{pH}$ sangüíneo entre 7,11 e 7,18, com exceção de um animal que foi tratado com lactato- $\mathrm{L}(\mathrm{pH}=6,88)$, e teores de bicarbonato menor que $20 \mathrm{mmol} / \mathrm{l}$ (Fig. 1). Em um dos animais tratados com lactato-L o $\mathrm{pH}$ do sangue chegou a atingir a preocupante marca de 6,88 no tempo zero, e mesmo assim, com uso apenas desse tampão, essa grave acidose foi corrigida, elevando o $\mathrm{pH}$ na terceira hora para 7,32 .

No momento basal não houve diferença significativa entre os animais tratados com bicarbonato como lactato-L em relação às variáveis $\mathrm{pH}$ e concentrações de bicarbonato, $\mathrm{TCO}_{2}$ e EAB (P>0,05) (Fig. 1 a 4$)$.

O pH sangüíneo foi sempre mais baixo no momento basal em relação aos demais tempos, tanto com o tratamento de bicarbonato como no de lactato-L $(\mathrm{P}<0,05)$. Maiores valores de $\mathrm{pH}$ sangüíneo foram encontrados da terceira a oitava horas em relação a primeira hora $(\mathrm{P}<0,05)$ entre os animais tratados com lactato-L (Fig. 1).

Não houve diferenças significativas $(\mathrm{P}>0,05)$ no $\mathrm{pH}$ sangüíneo dos animais medicados com as duas diferentes soluções no decorrer do experimento. Embora não significativo, os valores numéricos do $\mathrm{pH}$ sangüíneo dos bovinos tratados com lactato-L na oitava hora podem sugerir persistência de correção da acidemia, em relação aos tratados com bicarbonato (Fig. 1). O $\mathrm{CV}_{\mathrm{b}}$ do $\mathrm{pH}$ sangüíneo foi $5,2 \%$.

Em todos os animais tratados houve aumento, de forma semelhante, da concentração de bicarbonato sangüíneo nos vários tempos em relação ao momento zero. No tratamento com lactato-L isso ocorreu já na segunda hora pósinfusão $(\mathrm{P}<0,05)$ e assim se manteve até o final do experimento. No tratamento com bicarbonato, o aumento foi significativo a partir da terceira hora $(\mathrm{P}<0,05)$, ininterruptamente até a oitava hora (Fig. 2). $\mathrm{O} \mathrm{CV}_{\mathrm{b}}$ do teor de bicarbonato sangüíneo foi $8,5 \%$. 


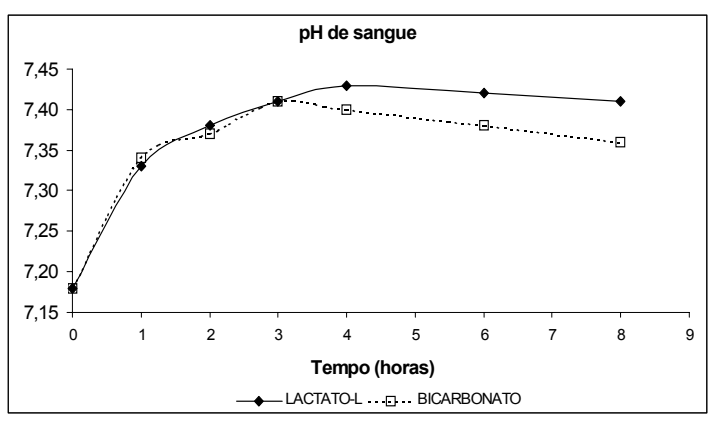

Figura 1. Evolução do pH sangüíneo durante a infusão de bicarbonato ou lactato-L, em bovinos com acidose láctica ruminal.

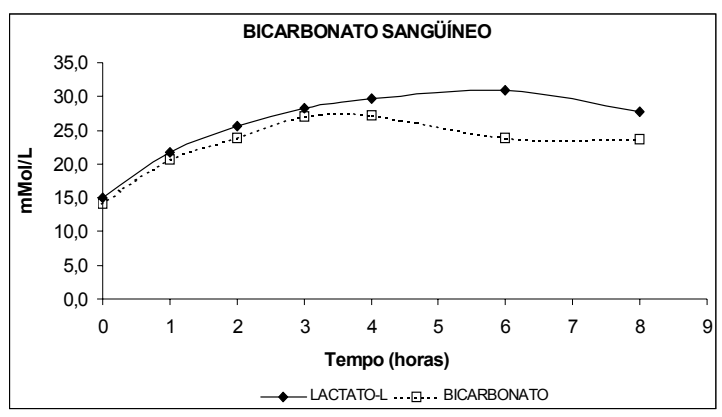

Figura 2. Evolução da concentração de bicarbonato sangüíneo $(\mathrm{mMol} / \mathrm{l})$ durante a infusão de bicarbonato ou lactato-L, em bovinos com acidose láctica ruminal.

Maiores concentrações de $\mathrm{TCO}_{2}$ e $\quad$ EAB sangüíneas ocorreram, de forma semelhante, ao longo do experimento, com ambos os tratamentos, em relação ao momento zero. No primeiro composto, houve aumento já na segunda hora pós-infusão; no tratamento com bicarbonato isso foi observado na terceira hora $(\mathrm{P}<0,05)$, e assim se manteve até o término do tratamento. No grupo infundido com bicarbonato, as elevações de $\mathrm{TCO}_{2}$ e EAB sangüíneas foram significativas da terceira a oitava horas $(\mathrm{P}<0,05)$ (Fig. 3 e 4$)$. Os $\mathrm{CV}_{\mathrm{b}}$ da concentração de $\mathrm{TCO}_{2}$ e EAB foram 9,3 e 12,4\% respectivamente.

Os diferentes tratamentos utilizados promoveram adequada recuperação clínica e não exigiram terapia adicional para correção de desidratação, ruminite ou normalização do $\mathrm{pH}$ do fluido ruminal. $\mathrm{O}$ apetite foi restabelecido

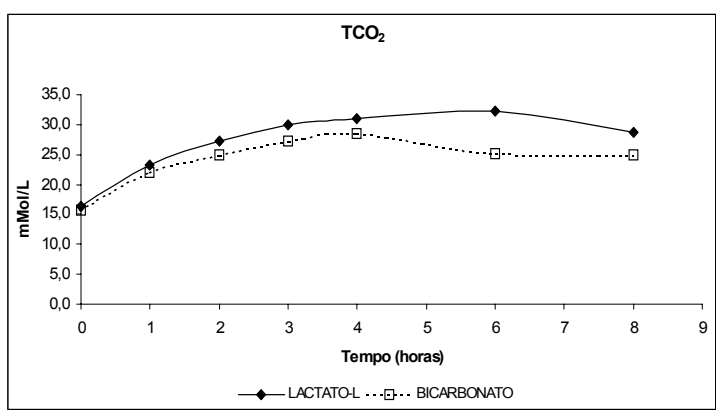

Figura 3. Evolução da concentração de $\mathrm{TCO}_{2}$ sangüíneo $(\mathrm{mMol} / \mathrm{l})$ durante a infusão de bicarbonato ou lactato-L, em bovinos com acidose láctica ruminal.

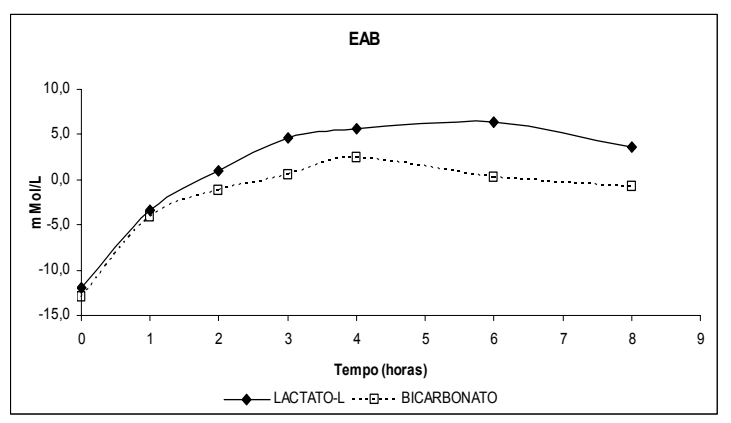

Figura 4. Evolução da concentração de excesso de ácido base $(\mathrm{EAB})$ sangüíneo $(\mathrm{mMol} / \mathrm{l})$ durante a infusão de licarbonato ou lactato-L, em bovinos com acidose láctica ruminal.

integralmente apenas no quarto dia, após o início do tratamento.

\section{DISCUSSÃO}

A quantidade global padronizada $(750 \mathrm{mmol} / \mathrm{l}) \mathrm{de}$ tampão empregado neste trabalho, independentemente do medicamento utilizado, foi suficiente para corrigir, dentro de três horas, a acidose metabólica sistêmica $(7,11$ a 7,18), classificada por Radostits et al. (2002) como de grande intensidade.

Tanto o pH como as reservas orgânicas de tampões (bicarbonato; $\mathrm{TCO}_{2}$ ) elevaram-se significativamente no decorrer do tratamento com lactato-L. Em relação ao tempo zero, esse tratamento, surpreendentemente, elevou as concentrações das reservas de tampões mais rapidamente - já na segunda hora - que o tratamento com bicarbonato - só a partir da 
terceira hora (Fig. 1 a 4). Embora não se tenha determinado o pH urinário, Mendes Netto (1997) observou que, após o tratamento de quadros de acidose láctica ruminal em bovinos, com infusão intravenosa de solução de bicarbonato, houve aumento posterior significativo no $\mathrm{pH}$ urinário, sugerindo que este foi causado pela excreção desse tampão na urina, o que não permitiu elevação demasiada de sua concentração no sangue. Fato idêntico foi verificado em bovinos sadios tratados com bicarbonato (Leal et al., 2006). Em nenhum animal Mendes Netto (1997) e Leal et al. (2006) detectaram quaisquer efeitos colaterais causados por uso excessivo de bicarbonato, como descrito por Hartsfield et al. (1981).

Embora muitos conceituem que a ação alcalinizante do lactato-L seja oriunda de sua transformação em bicarbonato (Hartsfield et al. 1981), essa afirmação é parcialmente verdadeira (Nelson e Cox, 2002). Grande parte desse efeito tampão vem do consumo de um íon $\mathrm{H}^{+}$quando da oxidação de uma molécula de lactato-L ou de sua transformação em glicose nos hepatócitos (Naylor e Forsyth, 1986): $2 \mathrm{CH}_{3} \mathrm{CHOHCOO}+$ $2 \mathrm{H}^{+}+6 \mathrm{O}_{2}=6 \mathrm{CO}_{2}+6 \mathrm{H}_{2} \mathrm{O}$ (oxidação) $2 \mathrm{CH}_{3} \mathrm{CHOHCOO}+2 \mathrm{H}+=\mathrm{C}_{6} \mathrm{H}_{12} \mathrm{O}_{6}$ (gliconeogênese).

No caso da oxidação, os produtos finais são $\mathrm{CO}_{2}$ e $\mathrm{H}_{2} \mathrm{O}$, os quais podem ou não, dependendo de uma série de condições bioquímicas, ser transformados em bicarbonato e íon $\mathrm{H}^{+}$, pela ação da anidrase carbônica (Nelson e Cox, 2002). Existindo esse consumo de $\mathrm{H}^{+}$, nessa reação oxidativa ocorrerá, concomitantemente, economia no bicarbonato sangüíneo, o qual será menos utilizado para tamponar o meio, aumentando sua concentração, como verificado no presente trabalho.

Diferente do que foi detectado em bezerros com diarréia por Case et al. (1980) e Kasari e Naylor (1985), o lactato-L promoveu excelente atividade alcalinizante em bovinos com ALR. Segundo esses autores, os animais com diarréia não conseguiram metabolizar adequadamente $\mathrm{o}$ lactato-L, em bezerros saudáveis, porém, tal processo ocorreu normalmente (Naylor e Forsyth, 1986). Segundo Case et al. (1980), bezerros com diarréia freqüentemente têm hipoglicemia e acidemia láctica e, nessas circunstâncias, embora a oxidação do lactato seja mantida, a geração de glicose a partir desse substrato é inibida. Por outro lado, ruminantes com ALR têm um quadro de hiperglicemia, sugerindo que a gliconeogênese, a partir do lactato, estaria aumentada (Angelov et al., 1995). Segundo Hartsield et al. (1981), não se recomenda o uso de lactato-L como alcalinizante se o $\mathrm{pH}$ sangüíneo for inferior a 7,1, encontrado em quadros de acidose severa, pois nessa condição ocorreria menor oxidação do lactato-L. Surpreendentemente, um animal com $\mathrm{pH}$ sangüíneo extremamente baixo $(6,88)$, quando do início do tratamento com lactato-L, corrigiu adequadamente o quadro de acidose em três horas de terapia $(7,32)$, colocando em dúvida tal conceito. Maiores estudos são necessários para confirmar a ação alcalinizante do lactato-L em condições críticas de acidose metabólica sistêmica.

Apesar dos resultados não serem significativamente diferentes $(\mathrm{P}>0,07)$, é importante frisar a tendência dos valores de $\mathrm{pH}$ e das concentrações de bicarbonato, $\mathrm{TCO}_{2}$ e $\mathrm{ABE}$ sangüíneos dos animais tratados com bicarbonato e lactato-L (Fig. 1 a 4). Enquanto que numericamente os valores máximos atingidos com a infusão de bicarbonato foram na terceira hora para o $\mathrm{pH}$ e na quarta para os demais metabólitos, o lactato-L promoveu esses picos na quarta e sexta horas, respectivamente, apresentando uma queda posterior mais lenta que o bicarbonato. Isso sugere que o lactato continua a ser metabolizado e apresenta ação alcalinizante mesmo depois do término da sua infusão.

\section{CONCLUSÕES}

O lactato-L pode e deve ser utilizado como uma alternativa ao tratamento clássico com bicarbonato, tendo a mesma eficiência que este último para a correção da acidose metabólica sistêmica causada pela ALR.

\section{AGRADECIMENTOS}

Os autores agradecem ao CNPq pelo auxílio financeiro para execução desta pesquisa e a bolsa de aperfeiçoamento concedida à primeira autora e as laboratoristas Clara Satsuki Mori e Regina Mieko Sakata Mirandola. 


\section{REFERÊNCIAS BIBLIOGRÁFICAS}

ANGELOV, G.; NIKOLOV, Y.; ANGELOV, A. Changes in acid-base variables and some biochemical parameters in caprine acute rumen acidosis. Vet. Arhiv., v.65, p.43-48, 1995.

ANUÁRIO DA PECUÁRIA BRASILEIRA, v.10, p. 5 e 88, 2003.

CASE, G.L.; PHILLIPS, R.W.; CLEEK, J.L. Lactic acid and glucose metabolism in healthy, lactic acid-infused, and diarrheic calves. Am. J. Vet. Res., v.41, p.1035-1038, 1980.

HARTSFIELD, S.M.; THURMON, J.C.; BENECH, G.J. Sodium bicarbonate and bicarbonate precursor for treatment of metabolic acidosis. J. Am. Vet. Med. Assoc., v.179, p.914916, 1981.

HOWARD, J.L. Ruminal metabolic acidosis. Bov. Pract., v.16, p. 45-63, 1981.

KASARI, T.R.; NAYLOR, J.M. Clinical evaluation of sodium bicarbonate, sodium Llactate, and sodium acetate for the treatment of acidosis in diarrheic calves. J. Am. Vet. Med. Assoc., v.187, p.392-397, 1985.

LEAL, M.L.R.; MORI, C.S.; ORTOLANI, E.L. Estudo comparativo da capacidade alcalinizante de tampões metabolizáveis em bovinos sadios. Arq. Bras. Med. Vet. Zootec., v.59, p.965-970, 2007.

MARUTA, C.A.; ORTOLANI, E.L. Suscetibilidade de bovinos das raças Jersey e Gir à acidose láctica ruminal: Acidose metabólica e metabolização do lactato-L. Cien. Rural., v.32, p.61-65, 2002.

MENDES NETTO, D. Comparação do uso de soluções de bicarbonato e ringer com lactato no tratamento da acidose metabólica de garrotes com acidose láctica ruminal aguda. 1997. 81f. Dissertação (Mestrado) - Faculdade de Medicina Veterinária e Zootecnia, Universidade de São Paulo, São Paulo.

NELSON, D.L.; COX, M.M. Lehninger princípios de bioquimica. 3.ed. São Paulo: Sorvier, 2002. 975p.

NAYLOR, J.M.; FORSYTH, G.W. The alkalinizing effects of metabolizable bases in the healthy calf. Can. J. Vet. Res., v.50, p.509-516, 1986.

ORTOLANI, E.L. Induction of lactic acidosis in cattle with sucrose: Relationship between dose, rumen fluid $\mathrm{pH}$ and animal size. Vet. Hum. Toxicol., v.37, p.462-464,1995.

RADOSTITIS, O.M.; GAY, C.C.; BLOOD, D.C. et al. Clínica Veterinária. Um tratado de doenças dos bovinos, ovinos, suínos, caprinos e eqüinos. 9.ed. Rio de Janeiro: Guanabara Koogan, 2002. $1737 \mathrm{p}$.

SAMPAIO, I.B.M. Estatística aplicada à experimentação animal. 2.ed. Belo Horizonte: Fundação de Ensino e Pesquisa em Medicina Veterinária e Zootecnia, 2002. 265p.

SUCUPIRA, M.C.A.; ORTOLANI, E.L. Uso do sangue arterial e venoso no exame do equilíbrio ácido-básico de novilhos normais ou com acidose metabólica. Cien. Rural, v.33, p.863868, 2003.

UNDERWOOD, W.J. Rumen lactic acidosis. Part II. Clinical signs diagnosis, treatment and prevention. Comp. Cont. Educ., v.14, p.12651270, 1992. 